Current Practice

\title{
SPECIAL SENSES
}

\section{Otosclerosis: A Synopsis of Natural History and Management}

\author{
ANDREW W. MORRISON,* F.R.c.s.
}

Otosclerosis is a common, hereditary disease of the temporal bone, found predominantly in the part surrounding the labyrinth and cochlea. Mature lamellar bone is removed by osteoclasis and replaced by new unorganized woven bone of greater thickness, cellularity, and vascularity, such as might be seen after a fracture or in response to infection or other trauma. The commonest site of origin of otosclerosis is in front of the oval window. The disease can also arise in the footplate of the stapes itself, in the region of the round window, and in other parts of the cochlear capsule.

An otosclerotic focus may cause no symptoms, its presence being detected at necropsy-this is referred to as histological otosclerosis. The focus may progressively affect the footplate of the stapes, giving rise to increasing fixation-clinical or stapedial otosclerosis, the variety most commonly encountered in practice. If otosclerosis affects the endosteal bone surrounding the cochlea or labyrinth it can give rise to secondary sensorineural changes, both cochlear and vestibular, and this is referred to as cochlear otosclerosis. The expression combined otosclerosis is used to signify a combination of stapedial and inner ear involvement.

The condition has been recognized for over 100 years. It is quite remarkable that before the end of the nineteenth century the stapes had been approached surgically and even removed. In those days there were no antibiotics, no prosthetics, no adequate anaesthesia, and no operating microscopes. It is not surprising that these early surgical attempts were forgotten for half a century. The prejudice against surgery was largely overcome when Lempert ${ }^{1}$ popularized the onestage fenestration operation in the late 1930s. In properly selected cases this operation gave fairly good results, but it had many disadvantages and has been discarded.

In the $1950 \mathrm{~s}^{2}$ there was a short era when mobilization of the stapes replaced the fenestration operation. The mobilization operation had several merits. It respected normal anatomy and physiology, it reintroduced the approach which forms the basis of today's lateral tympanotomy, and it was a stimulus for the development of the modern operating microscope. Only $20 \%$ of patients were helped.

The last 12 years $^{3}$ have seen the introduction and development of the modern surgical treatment of otosclerosisstapedectomy.

\section{Genetic Factors and Natural History}

Otosclerosis has been demonstrated in Homo sapiens only, and it is a very common cause of deafness in caucasian peoples throughout the world. It is less frequent in negroid and mongoloid man.

Clinical or stapedial otosclerosis causing conductive deafness affects about 4 per 1,000 of the adult population. The onset is normally between 15 and 45 years of age, with a

* Consultant E.N.T. Surgeon, the London Hospital and Royal National Throat, Nose and Ear Hospital, London. peak in the third decade. In clinical practice otosclerosis is encountered about twice as frequently in females as in males, but this does not reflect the true sex ratio. When families are investigated it is found that the condition is only slightly commoner in women, and, furthermore, unilateral deafness is much commoner in men. The patient with unilateral progressive deafness is less likely to seek medical advice.

There are other reasons for otosclerosis appearing to be more frequent in women. It has long been recognized that the deafness can be initiated or aggravated during pregnancy. This happens to just over $50 \%$ of affected women, mainly towards the end of pregnancy. It should also be noted that there are more females in the population under study. A characteristic of any conductive deafness is the ability of the patient to hear better in the presence of noise. This phenomenon, known as paracusis, is very typical of otosclerosis. The man, tending to work and live in a noisier environment, is less likely to be aware of his disability.

Upon adequate inquiry there are secondary cases in the family in $70 \%$ of patients, and the disease can be traced through several generations. In $30 \%$ of cases it appears to be sporadic. The condition has an autosomal dominant inheritance, the degree of manifestation varying from family to family, even this appearing to be genetically determined. Some families are encountered with $100 \%$ manifestation, others with $10 \%$. Almost half the individuals carrying the abnormal gene develop clinical otosclerosis.

Clinical severity, including unilateral involvement, varies from family to family, and this is most useful in assessing the prognosis when other family members are affected. In $85 \%$ of patients the progression of deafness is slow but relentless. It can best be measured in decades rather than years. After three or four decades the deafness is likely to be very severe, with secondary sensorineural loss. Less than $10 \%$ of patients are more fortunate, in that their hearing loss does not progress beyond the 50 to 60 decibel level. On the other hand, an equal number show rapid progression; this malignant otosclerosis is usually associated with an adolescent onset, early cochlear involvement, and sometimes a Schwartze sign. This is a pink tinge of the tympanic membrane seen on otoscopic examination and due to the reflection of red, vascular bone on the promontory.

With the passage of time the macroscopic thickening at the footplate becomes more severe. This has a direct bearing on the surgical treatment, which can be more difficult in longstanding cases.

These remarks on the inheritance and natural history also apply to pure cochlear otosclerosis, which is often familial, and where severity varies from family to family. When the inner ear is involved either in pure cochlear otosclerosis or combined with stapedial fixation the labyrinth can be affected. Some $5 \%$ of patients show vestibular symptoms, usually a benign, paroxysmal, positional vertigo with transient dizziness on lying down in bed or upon upward gaze. Occasionally, however, the attacks of vertigo last several hours and 
mimic. Ménière's disease. The frequency of inner-ear disease in otosclerosis is difficult to assess with accuracy. It seems likely that pure cochlear otosclerosis affects about 4 per 10,000 of the adult population. Combined otosclerosis is much more frequent - one third of patients with stapedial ankylosis eventually develop inner ear disease.

\section{Clinical Features}

The patient with stapedial otosclerosis complains of increasing bilateral deafness. The symmetry of the hearing loss is a characteristic feature, though $10 \%$ of female and $20 \%$ of male cases are predominantly unilateral. The phenomenon of paracusis has been mentioned. Another indication of conductive deafness during history taking is a quiet voice of good quality, which, being transmitted by bone conduction, is subjectively loud enough for the patient. Even when combined with sensorineural loss this quiet speech remains characteristic. Tinnitus is a definite complaint in a quarter of cases. It is encountered mainly in the older age group and in those with an early age of onset, and it often represents cochlear disease. When tinnitus is troublesome the patient frequently seeks advice regarding the outcome of surgery. If a good stapedectomy result is obtained it is likely that the patient will be less aware of head noises because of background masking, but there is no guarantee of this outcome.

On examination the tympanic membranes have a normal translucent appearance: The presence of minor scarring or atrophic change does not exclude the diagnosis, though it should raise the possibility of alternatives.

An assessment of the hearing loss using conversational and whispered voice or amplified speech together with the traditional tuning fork tests give helpful information on the degree of handicap. Though more sophisticated methods of investigation are available these simpler tests should not be ignored. The most reliable tuning fork for routine use is a small 256 with a large base for bone conduction. In stapedial otosclerosis the Rinne test is negative (bone better than air), and in unilateral or asymmetrical deafness the Weber is lateralized to the deafer side. The patient's bone conduction is normal (or even increased) compared with the examiner's. In pure cochlear otosclerosis the Rinne test is positive, the Weber lateralized to the better ear, and the bone conduction reduced. In combined otosclerosis the Rinne test is negative and the bone conduction reduced.

The pure-tone threshold for air and bone conduction is measured on a clinical audiometer. In early stapes fixation the air conduction graph is flat or gently rising, and in uncom-
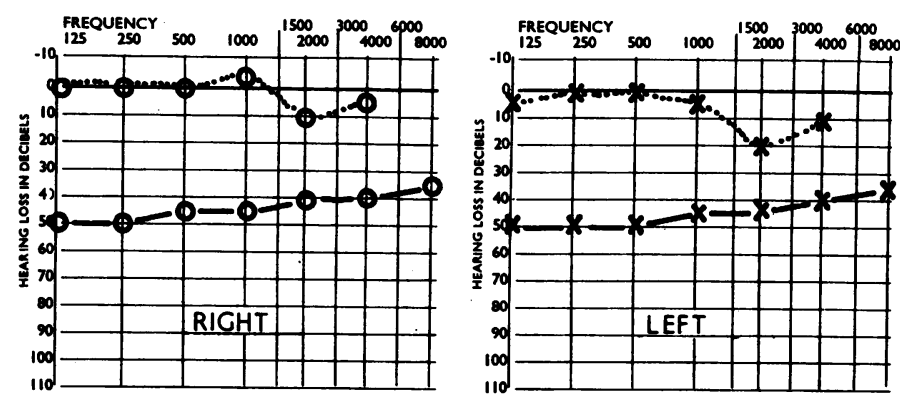

FIG. 1.-Audiogram in stapedial otosclerosis. Typical flat graphs with bone conduction notches at a frequency of $2000 \mathrm{~Hz}$.

plicated stapedial fixation this pattern persists with increasing ankylosis. The difference between the air and bone conduction levels is called the air-bone gap. This gap is of surgical signi ${ }^{1}$ cance-for, by and large, it represents the possible gain in hearing following stapedectomy. (Fig. 1).

A notch in the bone conduction at $2,000 \mathrm{~Hz}$. is considered another characteristic of stapedial otosclerosis. These notches are encountered in half the patients examined, and they probably represent evidence of inner ear disease. With the passage : of years the air and bone conduction levels tend to fall, and eventually in a small percentage of patients bone conduction is unrecordable. In these patients with total or subtotal deafness the Rinne test is still negative with the 256 fork (Fig. 2).

In cochlear otosclerosis without stapedial involvement the pure-tone audiogram often shows a notched or troughshaped loss. The other common finding is a high-tone deafness reminiscent of presbyacusis (Fig. 3). The age of onset, however, together with the family history, suggests the diagnosis. In this type of hearing loss the diagnosis can now
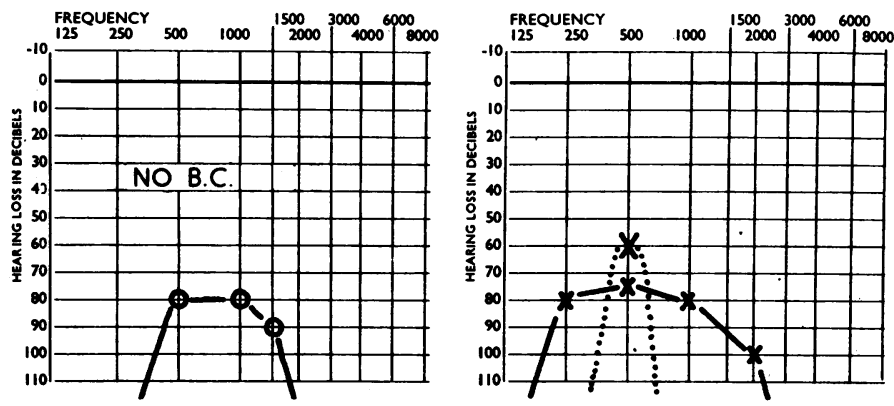

FIG. 2.-Audiogram in severe combined otosclerosis. Sub-total deafness. Rinne test negative both sides with 256 fork.
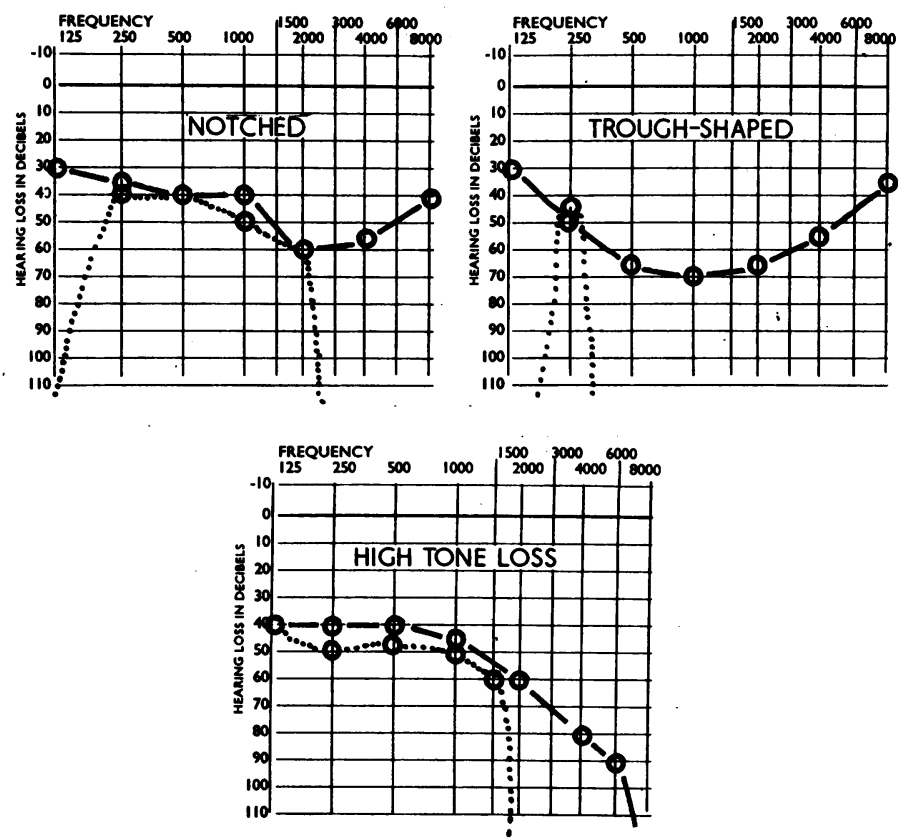

FIG. 3.-Audiograms in pure cochlear otosclerosis. Examples of sensorineural patterns in cochlear otosclerosis.

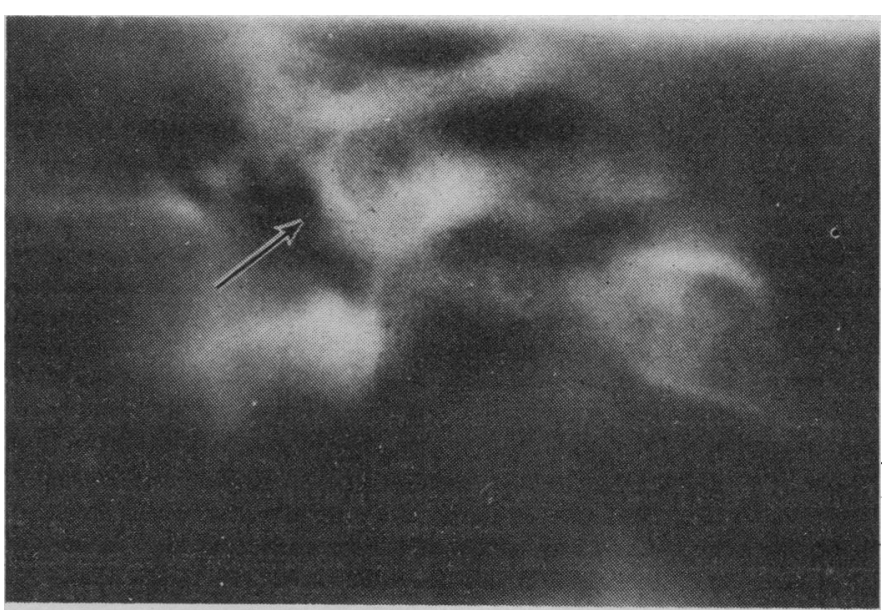

FIG. 4.-Polytome in cochlear otosclerosis. Radiolucency of basal coil. of cochlea (arrowed). Young man with positive Schwartze sign. 
be confirmed by polytomography. The radiographs show radiolucency over the promontory, the basal coil of the cochlea (Fig. 4).

\section{Specialized Tests}

In the majority of patients otosclerosis is a simple clinical diagnosis. For the occasional diagnostic problem or to assess the likely response to surgery in cases of combined otosclerosis a whole battery of specialized hearing tests is now available. These need not be described in detail, but it should be noted that the localization of the hearing loss can now be determined with a reasonable degree of accuracy at any given frequency.

The assessment of hearing loss by pure-tone or specialized audiometry does not always reflect the true handicap to the patient. Other factors have to be taken into consideration before advising surgical treatment. A moderate bilateral conductive deafness may be a distinct advantage to a patient working in a very noisy environment, whereas a unilateral conductive loss may be a great disadvantage to a musician or socialite.

\section{Differential Diagnosis}

Otosclerosis, with its varied clinical manifestations, can mimic a remarkable number of diseases causing both conductive and sensorineural deafness. It is important to make the correct diagnosis, since the surgical management of these conditions is different and stapedectomy is strongly contraindicated.

Other causes of ossicular immobility can be confused with stapedial otosclerosis. These include congenital ossicular fixations, the fixed malleus syndrome, tympanosclerosis, chronic adhesive otitis, chronic secretory otitis, and Paget's disease of bone. Paget's disease is worthy of special mention since a conductive deafness can be the only indication of the generalized bone disorder. The age of onset is usually over 45 .

Ossicular chain discontinuity occurs in congenital ossicular absence and after dislocation of the incus from head injury or old cortical mastoidectomy. Post-inflammatory loss of the long process of the incus is very common. Conductive deafness will also be encountered with chronic dry perforations and with chronic suppurative ear disease.

The diagnostic features and management of all these causes of conductive deafness cannot be covered in a brief review of this type. It is worth noting, however, that nowadays there is almost no cause of conductive deafness which cannot be cured, or at least improved, by surgical means.

\section{Treatment}

Surgery has no place in the management of pure cochlear otosclerosis. In combined otosclerosis a good stapedectomy result may leave the patient handicapped, even though there is closure of the air-bone gap. Sometimes operation is contraindicated in stapedial otosclerosis. The use of a transistorized hearing aid, with an insert air conduction receiver, gives excellent results in almost all these circumstances, provided speech discrimination is good. One aspect of prescribing an aid should not be forgotten- it does not prevent the natural progression of deafness.

Stapedectomy is contraindicated if the patient is unfit for surgery; it should never be carried out in children; it should never be performed on the patient's only hearing ear; it should be delayed if the hearing loss is minimal; and it should rarely be done in a previously fenestrated ear, since technical difficulties make for poor results. Operation is sometimes advised in unilateral fixation if the deafness is a real handicap.

\section{Stapedectomy}

The great majority of patients with stapedial or (s) ined otosclerosis derive benefit from surgical treatment. I. ormally the poorer hearing ear is chosen for surgery, which (in Britain) is performed under general anaesthesia. The patient is in hospital for three to six days.

With the aid of an operating microscope, through an aural speculum, a tympanomeatal skin flap is raised posterosuperiorly and the middle ear entered. This lateral tympanotomy (Fig. 5) gives an excellent view of the long process of the incus, the stapes, stapedius tendon, promotory, and round window. The facial nerve and chorda tympani are also visible. The chorda may have to be divided, but the patient rarely complains of the loca' zed loss of taste sensation.

The fixed stape, is next removed and replaced by a suitable prosthesis from incus to vestibule. There are numerous variations in operative technique at this stage of the reconstruction. Each has its ardent supporters and each gives good results in capable hands. Probably the Teflon piston is the simplest to use, though I prefer the stainless steel McGee piston and wire loop (Fig. 6).
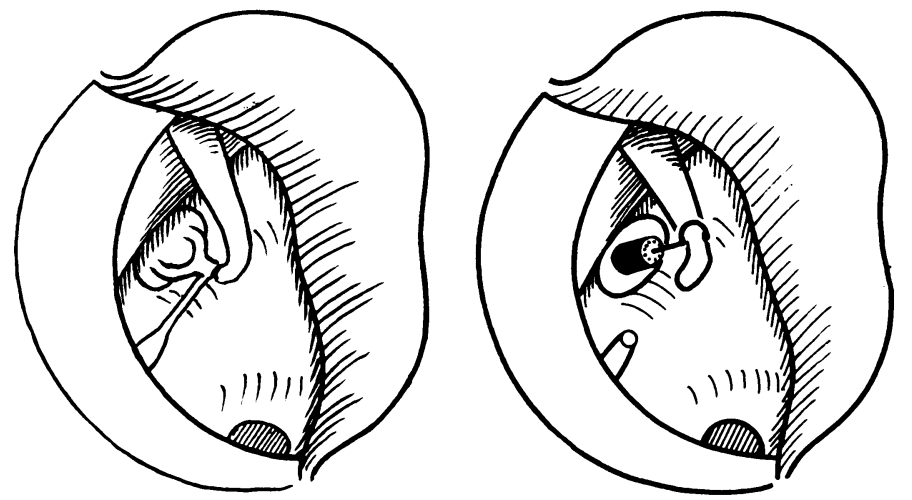

FIG. 5.- Lateral tympanotomy demonstrating facial nerve, long process of incus, stapes, stapedius tendon, promontory, and round window. Chorda tympani not shown. Fig. 6.--Stapedectomy: stapes removed and replaced by a stainless steel piston.

On completion of the operation the tympanomeatal skin flap is replaced, and a suitable dressing inserted into the external meatus for two to three days. During this time the patient may have transient unsteadiness induced by sudden head movements. The hearing may return at any stage from the early postoperative period to as late as several weeks. One week is about the usual.

\section{Results}

With current techniques $92 \%$ of patients can expect a good result-closure of the air-bone gap. Six per cent will be improved, though they will have failure of closure of the gap; and $2 \%$ are likely to have post-operative sensorineural loss. Over $90 \%$ of excellent results does not mean that all these patients have normal hearing. At least a third of patients have reduced bone conduction levels before surgery, and they have no prospect of ever attaining normal acuity. Many of them, however, manage without a hearing aid postoperatively if the air conduction level is raised to $30-40$ decibels. Even patients with total and sub-total deafness when first seen have a $50 \%$ chance of being able to hear with an aid after stapedectomy.

This operation must be classed as among the best in surgery. Experience from the last ten years suggests that provided hearing is maintained for four or five years it is likely to remain at a satisfactory level. After this interval of time stapedectomy may be considered on the opposite side.

In the early stapedectomy, era, when the stapes was replaced by a vein graft and polyethylene tube, postoperative 
conductive loss was not infrequently due to necrosis of the long process of the incus or disarticulation of the incudopolyethylene arthroplasty. These problems are rarely encountered with the prosthetics used today.

There are numerous explanations for the occasional postoperative sensorineural deafnesses. Sometimes it results from direct surgical trauma to the labyrinth due to technical difficulty. It is now recognized, however, that the commonest cause of both early and late sensorineural deafness is a perilymph fistula. After the footplate of the stapes has been removed nature has to provide a new thin membrane to which the prosthesis is loosely attached. This membrane normally forms during the first week or two, explaining the delay in improvement in hearing. Occasionally a fistula persists from the time of surgery, and in this event the patient will have fluctuating hearing, intermittent vertigo, failure of closure of the air-bone gap, and a high tone loss. This latter sensorineural element of ten gives poor speech discrimination. The signs, symptoms, and severity are directly related to the size of the fistula. If cerebrospinal fluid is flowing freely there is spontaneous vertigo and rapid sensorineural deafness. At the other extreme, a minute fistula will produce minimal symptoms and may heal spontaneously. After good results, perilymph fistulas have been encountered as late as six years. When the symptomatology suggests, a fistula re-exploration is indicated, sometimes urgently. Normally the original prosthesis has to be removed, the fistula has to be closed with a vein graft, and a new piston inserted.

Though a measure of standardization has been attained with otosclerotic surgery, the position is still slightly fluid. Much of the current-research and many of the minor operative modifications are currently directed towards avoiding these $2 \%$ of sensorineural problems.

\section{REFERENCES}

1 Lempert, J., Archives of Otolaryngology, 1938, 28, 42.

2 Rosen, S., Archives of Otolaryngology, 1952, 56, 610.

3 Shea, J. J., inr., Annals of Otology, Rhinology, and Laryngology, 1958, 67, 932.

\section{TODAY'S DRUGS}

With the help of expert contributors we print in this section notes on drugs in current use.

\section{Protection against Influenza}

Each year millions of people all over the world suffer from influenza, a small number die, and many millions of working hours are lost. Yet 37 years after the discovery of the first influenza virus ${ }^{1}$ we are still almost as powerless as then to prevent both epidemics and individual infections or to treat the illness with specific drugs. To understand the difficulties in dealing with this kind of problem we must know something about the causal viruses and the body's defences against invasion.

The two viruses responsible for epidemics of influenza are myxovirus influenzae type $A$ and myxovirus influenzae type $B$, influenza $A$ being the main culprit. It is a virus which shows major antigenic changes at approximately 10-year intervals, the last of these occurring in 1957 with the arrival of influenza A2, the Asian strain. In the periods between these major antigenic changes minor changes of antigenic structure occur, a phenomenon known as "antigenic drift." So after a time, though a strain of influenza may still be related to the original family prototype, considerable antigenic variation may have taken place. This is well illustrated by the current strain of influenza A2, the Hong Kong strain, which is still in the A2 or Asian family but sufficiently far removed to show little cross neutralization. Such variations affect the host's resistance or susceptibility to infection with the strain of influenza prevailing at the time.

Another important factor in resistance to influenza is the efficiency of antibody production and, in particular, the site at which antibody is produced. It it now recognized that in many respiratory infections the antibody produced in the respiratory tract bears a closer relationship to resistance than humoral antibody. ${ }^{23}$ This is of particular importance when attempts are being made to produce antibodies artificially by immunization. Despite the difficulties, some degree of protection against influenza is possible. This can be done in two ways, by use of vaccines or by drugs. Vaccines act by producing antibodies and drugs by preventing influenza virus infecting host cells.

\section{Vaccine Protection}

\section{Killed Vaccine}

The influenza virus antigen which appears to be responsible for inducing immunity is the haemagglutinin. In the past, trials with formalized influenza vaccine produced a high level of protection against infection by epidemic strains of virus of the same antigenic type if these were included in the vaccine. The apparent efficiency of a vaccine may be due to a number of factors. These include the accuracy of diagnosis of the respiratory illness from which the vaccinated patient may suffer; the antigenic type of virus in the vaccine compared to the one causing the epidemic; the timing of the vaccination programme in relation to the epidemic; and the power of the vaccine to produce high levels of antibody, particularly in the respiratory tract. It is therefore important to be careful in selecting the correct types of viruses to include in a killed vaccine. All are agreed that they should include influenza $A$ and $B$, but the type of influenza $A$ has been a matter of differing opinions. The Americans favour a mixture, including A, $A 1$, and A2. British workers have taken the view that the vaccine need only contain the latest variant of influenza $A$ and should be related as closely as possible to the epidemic strain. The formalized aqueous vaccine has the disadvantage of protecting only for a few months.

The next improvement was the discovery that aqueous suspensions of influenza vaccine could be mixed with mineral oil together with an emulsifying agent to produce a stable water-in-oil emulsion of low viscosity. ${ }^{4}$ Antibody produced with the oil-adjuvant influenza vaccine maintains a high level for at least a year.

The overall protection which killed influenza vaccines produce is in the neighbourhood of $60 \%-70 \%$. It is now believed that if the antigenic strain in the killed vaccine is closely related to the epidemic strain, a protective antibody will be produced; but when the antigenic difference is large, as exemplified by the change from $\mathrm{A} 1$ to $\mathrm{A} 2$ influenza or the appearance of the A2 Hong Kong, then the types of virus included in previous vaccines are no longer effective. Influenza vaccines are also not without their side-effects. Both aqueous and oil-adjuvant may cause pyrexial reactions due to impurities in the material used, and the latter may produce a local reactive nodule. It must also be borne in mind that these vaccines have been prepared in eggs, and though they contain only minimal amounts of egg protein after purification they should not be given to patients with known egg allergies, as severe reactions could be produced.

Attempts have been made more recently to extract the haemagglutinin from the whole virus particle and to use this as a purified vaccine. There are difficulties in its production, 